Physics Vol. 2, No. 5, pp. 209-215, 1966. Physics Publishing Co. Printed in Great Britain.

\title{
HIGH FREQUENCY LINEAR RESPONSE OF CLASSICAL FLUIDS
}

\author{
H. L. FRISCH \\ Bell Telephone Laboratories, Incorporated \\ Murray Hill, New Jersey \\ (Received 22 November 1965 )
}

\begin{abstract}
We investigate the asymptotic high frequency properties of the complex transport coefficients of a classical fluid in the long wavelength limit. Subject to assumptions corresponding essentially to the infinite differentiability intermolecular forces for real fluids we show that as $\omega-\infty$ the linear response of a classical fluid to a bulk periodic force is nondissipative. We investigate the effective order of magnitude of $\omega$ in order that such a response can be elucidated and diffusional, stress and temperature waves be observed. We conclude with a brief discussion of how these results must be modified for certain nondifferentiable intermolecular forces which arise in simplified statistical models of fluids.
\end{abstract}

THE low frequency, long-wavelength response of a classical fluid is governed by the laws of hydrodynamics. As the frequency increases it is expected that the hydrodynamic description will not be fully applicable, and it has often been modified to fit experimental data. We show that there exists one domain, that of very large (infinite) frequency $\omega$ and small enough (vanishing) wave number $k$, where precise statements can be made about the linear response of a fluid. In particular, we prove the existence of nondissipative high frequency modes, corresponding to temperature, diffusional and stress (standing) waves, which are the analogues for a classical liquid of second sound in He II. We outline briefly a possible experiment in which $k$ and $\omega$ are decoupled (so that we may be strictly in a low $k$, high $\omega$ regime), where these modes may be observable. We conjecture that it may be possible to observe similar effects in conventional experiments (e.g. Brillouin scattering in liquids), at high frequencies $\left(\sim 10^{9}-10^{10} \mathrm{c} / \mathrm{s}\right.$ ) where $\omega$ is not decoupled strictly from $k$, provided $k$ is less than a critical value.

We are concerned in this paper with the high frequency limiting behavior of the linear response of a classical fluid to a periodic (frequency $\omega$ ) bulk force. In the limit where the disturbances produced by the periodic force are of sufficiently long wavelength $(k-0)$. the response of the fluid is completely characterized by the appropriate complex $\omega$ dependent transport coefficient $[1,2,3]$,

$$
\begin{aligned}
\hat{\xi}(\omega) & =\lim _{\varepsilon \rightarrow 0+0} \int_{0}^{\infty} d t e^{+i \omega t} e^{-\varepsilon t}\langle J(0) J(t)\rangle \\
& =\xi(\omega)-i \mu(\omega) / \omega .
\end{aligned}
$$


In (1), $J(t)$ is a normalized tensor component of the microscopic flux of a suitable momentum corresponding to the bulk periodic force and the angular bracket denotes canonical averaging over an ensemble in the thermodynamic limit corresponding to the fixed number density of the fluid, $p$. The real part of $\hat{\xi}(\omega)$, is the frequency dependent, real transport coefficient $\xi(\omega)$, where

$$
\xi(\omega)=\int_{0}^{\infty} d t \cos (\omega t)<J(0) J(t)>
$$

(we suppress for simplicity in writing here and in what follows the $\varepsilon$ limit) and it characterizes the dissipative linear response of the system. The modulus function $\mu(\omega)$ is given by

$$
\mu(\omega)=\omega \int_{0}^{\infty} d t \sin (\omega t)<J(0) J(t)>,
$$

and it reflects the nondissipative response of the fluid.

In the case of a stress producing a pure shear or dilation, e.g., the appropriate complex transport coefficients $\hat{\xi}(\omega)$ are the complex shear and volume viscosities

$$
\begin{aligned}
& \hat{\eta}(\omega)=\eta(\omega)+i G(\omega) / \omega \\
& =\int_{0}^{\infty} d t e^{+i \omega t}<J^{x y}(0) J^{x y}(t)>, \\
& \hat{\eta}_{v}(\omega)=\eta_{v}(\omega)+i\left[K(\omega)-K_{0}\right] / \omega \\
& =\int_{0}^{\infty} d t e^{+i \omega t}<\frac{1}{9} \sum_{\alpha, \beta} J^{\alpha \alpha}(0) J \beta \beta(t)>
\end{aligned}
$$

with

$$
J \alpha \beta=(V k T)^{-1 / 2} \sum_{j=1}^{N}\left(\frac{p^{\alpha}{ }_{j} p_{j}}{m}+F^{\alpha}{ }_{j} r^{\beta}{ }_{j}\right)-\delta_{\alpha \beta}\left[p V+\frac{\partial p V}{\partial<E>}(E-<E>)\right]
$$

where $k$ is Boltzmann's constant, $T$ the temperature, $p_{j}^{\alpha}, \alpha=x, y, z$, is the $\alpha$-th component of the momentum of the $j$-th molecule whose corresponding component of the position is $r_{j}^{\alpha}$ and is acted upon by the total intermolecular force $F_{j}, p$ is the thermodynamic pressure and $E$ is the total energy of the fluid. $\eta(\omega), \eta_{v}(\omega), G(\omega), K(\omega)$ are the real, frequency dependent shear and volume viscosities; and shear and bulk moduli respectively while $K_{0}=-V(d p / \partial V) s$ is the adiabatic modulus [3]. Analogous relations hold for other than mechanical forces, the $\hat{\xi}(\omega)$ playing the role of a generalized, inverse susceptibility, $X(\omega)$. Unfortunately, we cannot invoke, in general, the Kramers-Kronig relations to relate the real and imaginary parts of $X(\omega)$ since the half plane Im $\omega<0$ may contain, in general, singularities.

We can obtain asymptotic estimates as $\omega \rightarrow \infty$. For example if 


$$
R(t)=\langle J(0) J(t)\rangle
$$

possesses $N$ derivatives $R^{(n)}(t), n=1, \ldots, N$ in $(0, \infty)$ and $R^{(N)}(t)$ is integrable in $(0, \infty)$ then $[4]$

$$
\hat{\xi}(\omega) \tilde{a}-\sum_{n=0}^{N-1} i-n-1 \omega-n-1 R(n)(0)+o(\omega-N)
$$

as $\omega \rightarrow \infty$. Actually much more can be said. We note that subject to the usual hypothesis of statistical mechanics $J(t)$ is a (wide sense) stationary, ergodic process with the real covariance function

$$
\begin{aligned}
R(t)=\langle J(0) J(-t)\rangle & =\lim _{T \rightarrow \infty} \frac{1}{2 T} \int_{-T}^{T} d t_{0} J\left(t_{0}\right) J\left(t_{0}+t\right) \\
& =R(-t),
\end{aligned}
$$

or at worst is a convex combination of real covariance functions. In the latter case we can deal with each term in the combination separately, hence without loss of generality, $R(t)$ can be taken to be a single real covariance function in what follows.

The $J(t)$ are in general infinitely differentiable functions of the positions, momenta and intermolecular forces and are thus infinitely differentiable functions of the time if the timeindependent intermolecular forces are infinitely differentiable functions with respect to their arguments (which may be positions or momenta), by virtue of Hamilton's equations. This suggests the hypothesis that for real fluids with infinitely differentiable intermolecular forces, $R(t)$ is an infinitely differentiable function of $t$. We call this Assumption A. It follows immediately from Assumption A that for any fixed $N$,

$$
R^{(2 n+1)}(0)=0 \text { for all } n \text {, }
$$

that (6) reduces to

$$
\hat{\xi}(\omega) \tilde{a} \frac{i}{\omega} \sum_{n=0}^{N-1}(-1)^{n} \omega^{-2 n} R^{(2 n)}(0)+o\left(\omega^{-2 N+1}\right) \tilde{a} i \mu(\omega) / \omega
$$

as $\omega \rightarrow \infty$, i.e. (9) is an asymptotic expansion for the modulus $\mu(\omega)$ and for any $n<N$

$$
\omega^{2 n} \xi(\omega) \tilde{a} 0 \quad \text { as } \omega \rightarrow \infty \text {. }
$$

Furthermore it can be shown that the moments of $\xi(\omega)$ exist [5], i.e.

$$
M_{n}=\int_{0}^{\infty} d \omega \omega^{n} \xi(\omega)<\infty .
$$

A much more special set of cases correspond to Assumption B: That $R(t)$ considered as a function of the complex variable $t$ is analytic in the circle $|t|<\tau_{0}$ enclosing the origin. It follows that $R(t)$ is then analytic within a strip $|\operatorname{Im} t|<\tau_{0}$ about the whole real $t$ axis [6]. Equations (9) and (10) still apply and (11) can be replaced [7] by the exponential moment condition 


$$
\hat{M}(\tau)=\int_{0}^{\infty} e^{|\omega| \tau} \xi(\omega) d \omega<\infty
$$

for all positive $\tau<\tau_{0}$. It can be shown that Assumption B implies that with probability 1 a sample function $J(t)$ of the real variable $t$ is analytic, more precisely $J(t)$ is determined throughout its strip of regularity by analytic continuation of its values on any $t$ set which has a finite accumulation point in the strip of regularity [8]. For any realistic intermolecular potential it is hard to believe that this can be true.

Thus for fluids satisfying either Assumption A or B the linear response in the limit $\omega \rightarrow \infty$ $(k-0)$ is wholly nondissipative with

$$
\mu(\omega) \tilde{a} R(0)-\frac{R^{(2)}(0)}{\omega^{2}}+o\left(\omega^{-3}\right), \quad R(0)=\mu(\infty),
$$

in contradistinction to their response in the $\omega-0$ limit where it is wholly dissipative and described by the zero frequency transport coefficient, $\xi(0)$. The behavior of $\xi(\omega)$ as $\omega \rightarrow \infty$ is characterized by (10) and (11) or (12) depending on whether Assumption A or B applies. We can ask at what frequencies $\omega$ is the nondissipative limit as $\omega-\infty$ response of the fluid effectively attained? A rough estimate is given by virtue of (10) and (13) by the condition

$$
\omega \gg \sqrt{\left|\frac{R^{(2)}(0)}{R(0)}\right|}
$$

To consider a concrete example of this limiting behavior let us examine self-diffusion, for a fluid whose intermolecular forces are derivable from an (infinitely) differentiable potential $\phi=\phi(r)$ with $r$ the intermolecular separation. Consider a gaseous mixture of two isotopes which is sufficiently attenuated so that isothermal self-diffusion is uncoupled from any other transport process. In principle, if not in practice, one could excite isotope concentration oscillations which as $\omega \rightarrow \infty$ would properly be called undamped diffusional waves "propagating" as a forced mode with an apparent "velocity"

$$
v_{D}=\sqrt{\mu_{D}(\infty)}=\sqrt{\left\langle J_{D}(0) \cdot J_{D}(0)\right\rangle}=\sqrt{\left.\frac{1}{3}<\frac{p_{1}}{m} \cdot \frac{p_{1}}{m}\right\rangle}=\sqrt{\frac{k T}{m}}
$$

The gas molecules oscillate then along linear trajectories at thermal speed. Clearly this can only happen at frequencies $\omega$ larger than an inverse collision time. According to (14) the exciting frequency

$$
\omega>\sqrt{\frac{2 \pi \rho}{3 m} \int_{0}^{\infty} d r \frac{d}{d r}\left(r^{2} \frac{d \Phi}{d r}\right) g_{2}(r, \rho)}=\omega_{D}
$$

where $g_{2}(r, \rho)$ is the radial distribution function. In a dilute gas, $g_{2}$ is effectively zero for $r<\sigma$, the Lennard-Jones distance parameter, and unity for $r>\sigma$ so that with $\phi=4 \varepsilon\left[(\sigma / r)^{12}-\right.$ $\left.(\sigma / r)^{6}\right]$ one estimates $\omega_{D} \sim 16 \pi \varepsilon \sigma \rho / m$ or about $5 \times 10^{12} \mathrm{c} / \mathrm{s}$ for argon at N.T.P. This is well above a presently realizable frequency in a molecular system. 
Similarly there exists in principle the possibility of exciting shear waves in fluids. Earlier and independently Zwanzig and Mountain [3] found, cf. (4a-4c),

$$
\begin{aligned}
\mu_{\eta}(\infty) & =\left\langle\left(J^{x y}(0)\right)^{2}\right\rangle \\
& =\rho k T+\frac{2 \pi \rho^{2}}{15} \int_{0}^{\infty} d r \frac{d}{d r}\left(r^{4} \frac{d \phi}{d r}\right) g_{2}(r, \rho)=G(\infty)
\end{aligned}
$$

which except for the kinetic term $p k T$ is identical with the results of Green [9] and Fisher [10]. These authors [3] also have tabulated the reduced elastic moduli as a function of reduced density and temperature and derived (for a Lennard-Jones potential) a generalized Cauchy identity. Presumably these waves again become undamped for

$$
\omega>\sqrt{<J^{x y}(0) J x y(2)(0)>/ G(\infty)}=\omega_{\eta} .
$$

Temperature waves can also be excited in principle. If one neglects any coupling of heat conduction to other transport processes the "velocity" of these waves would be

$$
v_{T}=\sqrt{\frac{\mu_{T}(\infty)}{\rho c_{v}}}
$$

where $c_{v}$ is the specific heat at constant volume and

$$
\mu_{T}(\infty)=\left\langle J_{h}(0) \cdot J_{h}(0)\right\rangle
$$

where $J_{h}$ is the microscopic (dynamical) heat flux which enters the zero frequency heat conductivity, viz.

$$
\lambda(0)=\int_{0}^{\infty} d t<J_{h}(0) \cdot J_{h}(t)>
$$

For $\omega<\omega_{T}=\sqrt{<J_{h}(0) \cdot J_{h}{ }^{(2)}(0)>/ \mu_{T}(\infty)}$ these waves, even if they can be excited, would be presumably damped by the thermal conductivity, etc.

Possibly vestiges of these waves can be seen at physically realizable very high frequencies. Note that in view of our $(k-0)$ long wavelength limit, as already pointed out by Zwanzig and Mountain [3], experiments would have to be carried out in such a manner that the coupling of wavelength and frequency scales does not occur. In a polarizable fluid or suitable fluid mixture, density waves can be excited for example by electrostriction when the fluid is immersed in the cavity of a travelling wave tube for which $\omega$ and wave vector are not tied together [11]. Even the linearized hydrodynamic equations couple mass, momentum and energy transport so that a complete analysis of all density propagation modes, if this could be carried out experimentally, could reveal a multiplicity of characteristic velocities corresponding, e.g. to a "temperature wave". In principle at least, for $\omega \gg \omega_{T}$, classical fluids could exhibit undamped temperature waves somewhat reminiscent of second sound.

In conventional experiments, $\omega$ not decoupled from $k$, (e.g. Brillouin scattering [12-14]) one may conjecture that vestiges of these high frequency, yet $\omega<\omega_{D}$, modes can exist in 1 iquids 
away from phase transitions if the wave vector $k$ is less than a critical value, corresponding order of magnitude wise to a few multiples of a molecular diameter (say $k$ less than $k_{c} \sim$ $\left.10^{?} \mathrm{~cm}^{-1}\right)$. The temperature and stress modes, unlike the diffusional mode, are mediated by potential as well as kinetic contributions. It appears plausible, although we cannot sketch a proof, that vestiges of such modes persist through the "time-duration" of a "collision" in a dense fluid rather than a "hard core" collision time. Thus the frequencies above which one may begin to observe a temperature or stress mode in a dense fluid may be significantly lower than $\omega_{D}$, perhaps as low as $10^{9}-10^{10} \mathrm{c} / \mathrm{s}$. This may mean that in the Brillouin scattering experiments $[12-14]$ one is effectively entering a high frequency, low $k$ regime $\left(\omega \widetilde{>} \omega_{\hat{\eta}}, k<k_{c}\right)$.

A number of statistical mechanical models of simple fluids exist for which the intermolecular force is not infinitely differentiable, e.g. fluids with "hard cores" the simplest case being the hard sphere fluid. Assumption A fails but in general for $t \rightarrow 0$,

$$
R(t) \tilde{a} 1-\beta|t|+0(|t|) \text {. }
$$

Asymptotically as $\omega \rightarrow \infty,(10)$ no longer applies but instead

$$
\xi(\omega) \tilde{a} \frac{\beta}{\omega^{2}}
$$

as $\omega \rightarrow \infty$. Furthermore for those transport coefficients for which $J$ contains the intermolecular force in a linear combination, as in the viscosities or thermal conductivity, the corresponding modulus function $\mu(\infty)$ becomes indefinitely large. This is an immediate physical consequency of the fact that there is a finite fraction of molecules in contact. This is easily seen from (17). Consider in (17) the repulsive potential. $\phi(r)=\varepsilon(\sigma / r)^{n}$ which approximates the hard sphere potential as $n^{-1}-0$, with hard sphere diameter $\sigma$. We write, following Rowlinson [12],

$$
g_{2}(r, \rho)=y_{n}(r, \rho) \exp [-\phi(r) / k T]
$$

in (17) noting that $y_{n}$ is a smooth function of $r, \rho$ and $T$ even at $r=0$ as $n^{-1} \rightarrow 0$. We find that as $n^{-1} \rightarrow 0$,

$$
\frac{G(\infty)}{n-3} \tilde{a} \frac{2 \pi \rho^{2} \sigma^{3} \varepsilon y_{\infty}(\sigma)}{15}\left(\frac{\varepsilon}{k T}\right)^{3 / n-1} \Gamma\left(1-\frac{3}{n}\right)
$$

where

$$
\frac{2 \pi}{3} \rho \sigma^{3} y_{\infty}(\sigma)=\frac{p_{h}}{\rho k T}-1
$$

with $p_{h}$ the pressure of a hard sphere assembly of density $p$ at temperature $T$.

\section{Acknowledgment}

We are indebted in the course of this work to fruitful discussions with P. Hohenberg, N. Gillis, F. Stillinger, P.W. Anderson and C. Herring. 


\section{References}

1. P.C. MARTIN, Non-Local Transport Coefficients Correlation Functions, in Statistical Mechanics of Equilibrium and Non-Equilibrium, J. Meixner, ed. North Holland (1965).

2. J.A. MCLENNAN, Phys. Fluids 3, 493 (1960).

3. R. ZWANZIG and R.D. MOUNTAIN, preprint entitled "High Frequency Elastic Moduli of Simple Fluids" .

4. A. ERDÉLYI, Asymptotic Expansions, p. 47. Dover Publications (1956).

5. M. LOÈVE, Probability Theory, p. 199. D. Van Nostrand (1955).

6. Ibid., p. 212 .

7. Ibid., p. 213 .

8. Yu.K. BELAYEV, Teor. Veroyatnost. i Primen, 4, 437 (1959) also S.P. Lloyd, unpublished.

9. H.S. GREEN, The Molecular Theory of Fluids, p. 181. North Holland (1952).

10. I.Z. FISHER, Statistical Theory of Liquids, p. 60. University of Chicago Press (1964).

11. F.S. STILLINGER, private communication.

12. I. L. FABELINSKII, Sov. Phys. Usp. 5, 667 (1963).

13. R. Y. CHIAO and B.P. STOICHEFF, J. Opt. Soc. Am. 54, 1286 (1964); G. B. BENEDEK, J.B. LASTOVAK, K. FRITSCH and T. GREYTAK, $i b i d, 54,1284$ (1964).

14. P. A. FleURY V, Quart. Prog. Rept. Res. Lab. Electr. MIT No. 78, p. 80 (1965).

15. J.S. ROWLINSON, Mol. Phys. 8, 107 (1964). 